\title{
A Spontaneous Thoracic Spinal Epidural Hematoma Causing Hemiplegia and Back Pain
}

\author{
Bedia Gülen', Tolga Turan Dündar², Ertan Sönmez', Güleser Akpınar ${ }^{3}$ \\ 'Department of Emergency Medicine, Bezmialem Vakıf University School of Medicine, İstanbul, Turkey \\ 2Department of Neurosurgery, Bezmialem Vakıf University School of Medicine, İstanbul, Turkey \\ ${ }^{3}$ Clinic of Emergency Medicine, Şişli Etfal Training and Research Hospital, İstanbul, Turkey
}

\begin{abstract}
Spontaneous spinal epidural hematoma (SEH) is an uncommon and emergency condition leading to spinal cord compression. Early diagnosis can be achieved by magnetic resonance imaging (MRI) or computed tomography, but preferably MRI. Surgical decompression is urgent and can relieve neurologic deficits. A 27-year-old man presented to the emergency department with back pain, which began approximately 10 days before. While waiting for the results of biological markers for differential diagnosis, right hemiplegia became evident. Epidural hematoma was revealed with thoracic and lumbar MRI at level T1-T2. Hematoma evacuation and decompression laminectomy was immediately performed.

Keywords: Spinal epidural hematoma, back pain, hypoesthesia
\end{abstract}

\section{Introduction}

Spontaneous spinal epidural hematoma (SEH) is described as a spontaneous collection of blood in the spinal epidural space and often causes devastating sequelae unless it is diagnosed in time and effectively managed. Incidence rate is estimated at 0.1 per 100,000 individuals. Studies show that most cases occur in $>40$ year-olds $(1,2)$.

Ethiopathogenesis is usually related to neoplasms, coagulopathy, vascular malformation, traumas, drugs; however, in up to $40 \%$ cases, no underlying cause is identified. SEH mostly presents as acute to subacute pain at the level of the spinal hemorrhage, and neurological symptoms may develop including sensory disturbance, progressive weakness, urine and stool retention or incontinence, and paralysis. Atypical SEH may appear as an acute ischemic stroke or myocardial infarction (3). We report a case of thoracic spinal epidural hematoma associated with acute hemiplegia, which was treated with decompression with $100 \%$ recovery.

\section{Case Presentation}

A 27-year-old man presented to the emergency department with back pain, which began about approximately 10 days before.
The patient's temperature was $36.5 \mathrm{C}^{\circ}$, heart rate was 68 beats/ min, blood pressure was $126 / 72 \mathrm{mmHg}$, respiratory rate was 18 breaths/min, and oxygen saturation was $99 \%$ on room air. The patient was alert, oriented, and cooperated. The cardiovascular, pulmonary, and abdominal examinations were unremarkable. Examination findings included thoracic and lumbar sensitivity. There was no past medical history or trauma. While waiting for the results of biological parameters for differential diagnosis, right hemiplegia occurred. Weakness in the right leg developed within the first hour following admission. There were no abnormal findings on laboratory or bidirectional lumbar x-ray graphics. Neurological findings of the patient consisted of a normal cranial nervous system with rapid progression of weakness of the right lower extremity from grade $1 / 5$ to grade $2 / 5$. Deep tendon reflexes decreased with time. Hypoesthesia developed at T10. Cranial, cervical, thoracic, and lumbar magnetic resonance imaging (MRI) were planned after these complaints and findings. Thoracic MRI showed a T1 isointense (Figure 1) and T2 hyperintense (Figure 2) mass lesion in the posterior epidural space. The patient was subsequently hospitalized in the neurosurgery clinic. Hematoma evacuation and decompression laminectomy were immediately performed. The patient was discharged from the hospital on post-operative day 9 with total recovery. 


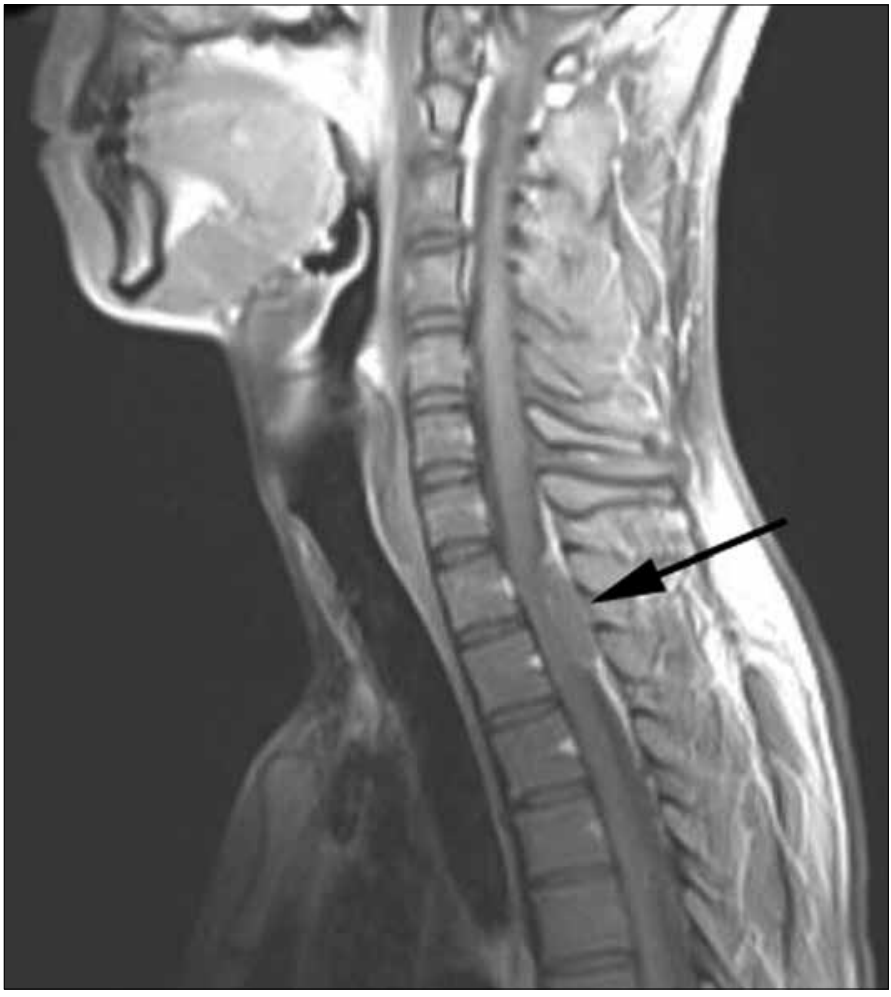

Figure 1. Contrast enhanced T1-weighted sagittal image showing the isointense posterior epidural mass lesion, without contrast enhancement, that compressed the spinal cord at the level of thoracic $1-2$ vertebrae (black arrow)

\section{Discussion}

Spontaneous SEH is an important clinical presentation for neurological emergency. Spontaneous SEH may appear due to epidural venous plexus favored by coagulation, hemangiomas, or spinal arteriovenous malformations (4).

Early detection of spontaneous SEH is difficult process due to atypical symptoms. Clinical symptoms suddenly occur, and neurological symptoms may vary (5). Most researchers claim that it occurs at the epidural venous plexus in the spinal epidural space of the thoracic spine, and the abdominal cavity can also be directly affected. However, Miyagi et al reported that arterial blood supply is a more persuasive force for pressure inside the venous plexus as it is lower than that in the epidural space. Furthermore, in some cases, rapidly deteriorating neurological deficits after the initial onset of back pain or lumbago indicated a rapid formation of the hematoma and spinal cord compression, which is suggestive of the arterial origin of spontaneous SEH (6).

Prompt diagnosis is difficult due to atypical symptoms, variations in age, and clinical suspicion is also difficult. Rapid diagnosis by $\mathrm{MRI}$ is pivotal because neurological symptoms are often severe (7). Although epidural hematomas are usually found on the posterior spinal cord in a lentiform shape, spontaneous SEHs are commonly observed on the anterolateral and around the spinal cord in a semi-circular pattern, accompanied with compression and some displacement (8).

Spontaneous SEH should be routinely considered in patients who have symptoms of spinal cord compression without other clinical findings. Spinal masses are frequently noticed during differential diagnosis. Definitive diagnosis is mostly achieved with operation.

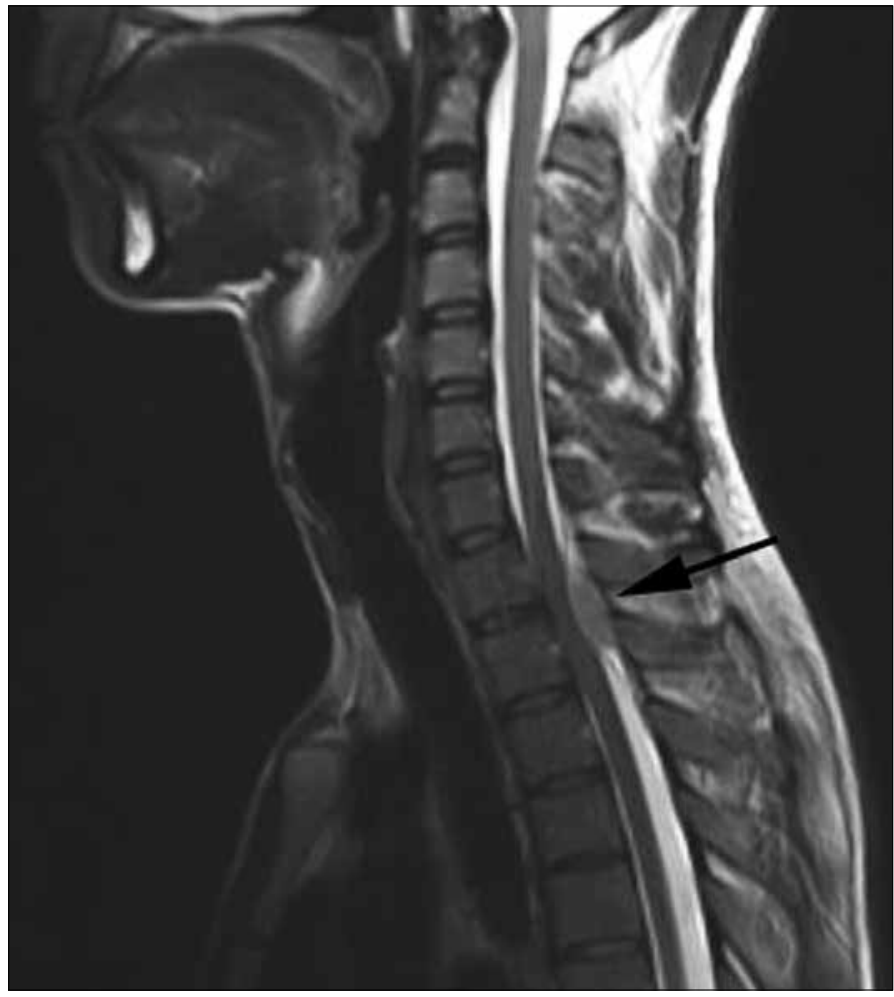

Figure 2. T2-weighted sagittal image showing the hyperintense posterior epidural mass lesion that compressed the spinal cord at the level of thoracic 1-2 vertebrae (black arrow)

Not only are the findings of spontaneous SEH seen; furthermore, pathological evaluation is also required to diagnose lymphoma and metastasis. In addition, MRI findings of these conditions are similar to those of spontaneous SEH. Spontaneous SEH mostly occurs in the thoracic spine, usually extending over several vertebrae. The pathological specimen in our case was a hematoma. Surgical removal of the hematoma was inevitable in this case, although conservative treatment may be sufficient in some cases (9).

Our patient suffered from lumbar pain for approximately 10 days. Neurological examination was normal during the first evaluation of the patient. Oversensitivity of the spinal process and paravertebral line of the thoracic vertebrae was established. However, SEH should be considered by emergency physicians in the pre-diagnosis of patients with a variety of neurological complaints. Fortunately, the rapidly deteriorating neurological deficits during observation and the subsequent emergency neurosurgery intervention ensured smooth recovery for our patient.

Spontaneous SEH should be suspected in patients admitted with a common back pain in crowded emergency departments. Early diagnosis and timely management of spontaneous SEH may improve prognosis and outcome. In this case, MRI was vitally important as the patient presented with neurological symptoms following back pain.

Informed Consent: Written informed consent was obtained from patient who participated in this case.

Peer-review: Externally peer-reviewed.

Conflict of Interest: No conflict of interest was declared by the authors. 
Financial Disclosure: The authors declared that this study has received no financial support.

\section{References}

1. Liu Z, Jiao Q, Xu J, Wang X, Li S, You C. Spontaneous spinal epidural hematoma: analysis of 23 cases. Surg Neurol 2008; 69: 253-60. [CrossRef]

2. Rajz G, Cohen JE, Harnof S, Knoller N, Goren O, Shoshan Y, et al. Spontaneous spinal epidural hematoma: the importance of preoperative neurological status and rapid intervention. J Clin Neurosci 2015; 22: 123-8. [CrossRef]

3. Estaitieh N, Alam S, Sawaya R. Atypical presentations of spontaneous spinal epidural hematomas. Clin Neurol Neurosurg 2014; 122: 135-6. [CrossRef]

4. Saritas A, Guneysu F, Guneysu S, Buyukkaya, Kandis H. An unusual presentation of spontaneous spinal epidural hematoma. J Emerg Med 2014; 47: 689-91. [CrossRef]
5. Schatlo B, Panciani PP, Bijlenga P, Schaller K. Cyclic alternation of paraplegia and leg pain as symptoms of a giant spontaneous spinal epidural haematoma. Acta Neurochir 2014; 156: 2155-7. [CrossRef]

6. Tiryaki M, Basaran R, Aydin SO, Efendioglu M, Balkuv E, Balak N. Spontaneous cervical epidural hematoma with hemiparesis mimicking cerebral stroke. Case Rep Emerg Med 2014; 2014: 210146. [CrossRef]

7. Felton B, DaoT, Gerstner B, Letarte S. Diagnosis of spinal epidural abscess by abdominal plain-film radiography. West J Emerg Med 2014; 15: 885-6. [CrossRef]

8. Kim T, Lee CH, Hyun SJ, Yoon SH, Kim KJ, Kim HJ. Clinical outcomes of spontaneous spinal epidural hematoma: a comparative study between conservative and surgical treatment. J Korean Neurosurg Soc 2012; 52: 523-7. [CrossRef]

9. Payer M, Agosti R. Spontaneous acute spinal subdural hematoma: spontaneous recovery from severe paraparesis--case report and review. Acta Neurochir 2010; 152: 1981-4. [CrossRef] 